\title{
Multiple Fano resonances with flexible tunablity based on symmetry-breaking resonators
}

\author{
Xiao bin Ren ${ }^{1}$, Kun Ren ${ }^{*}$, Ying Zhang ${ }^{2}$, Cheng guo Ming ${ }^{1}$ and Qun $\mathrm{Han}^{2}$
}

\author{
Full Research Paper \\ Address: \\ ${ }^{1}$ School of Science, Tianjin University of Science and Technology, \\ Tianjin 300222, China and ${ }^{2}$ College of Precision Instrument and \\ Opto-electronics Engineering; Key Laboratory of Opto-electronics \\ Information Technology, Ministry of Education, Tianjin 300072, China \\ Email: \\ Kun Ren ${ }^{*}$ - renkun@tju.edu.cn \\ * Corresponding author

\section{Keywords:} \\ multiple Fano resonance; off-centered ring resonators; plasmonic \\ waveguide; surface plasmon polaritons; symmetry-breaking; tunable \\ resonance
}

Beilstein J. Nanotechnol. 2019, 10, 2459-2467. doi:10.3762/bjnano.10.236

Received: 27 June 2019

Accepted: 12 November 2019

Published: 11 December 2019

Associate Editor: P. Leiderer

(C) 2019 Ren et al.; licensee Beilstein-Institut. License and terms: see end of document.

\begin{abstract}
A symmetry-breaking nanostructure is proposed to achieve multiple Fano resonances. The nanostructure consists of an asymmetric ring resonator coupled to a plasmonic waveguide. The broken symmetry is introduced by deviating the centers of regular ring. New resonant modes that are not accessible through a regular symmetric ring cavity are excited. Thus, one asymmetric cavity can provide more than one resonant mode with the same mode order. As a result, the interval of Fano resonances is greatly reduced. By combining different rings with different degrees of asymmetry, multiple Fano resonances are generated. Those Fano resonances have different dependences on structural parameters due to their different physical origin. The resonance frequency and resonance peak number can be arbitrarily adjusted by changing the degree of asymmetry. This research may provide new opportunities to design on-chip optical devices with great tuning performance.
\end{abstract}

\section{Introduction}

Fano resonances originate from the interference of a discrete state and a continuum state $[1,2]$. Contrary to symmetric Lorentz line shapes, a Fano profile is sharp and asymmetric. Due to this unique line shape and the large induced field enhancements, Fano resonances can potentially applied in sensors [3,4], demultiplexers [5], lasers [6], filters [7], and photoswitches [8].

Various structures have been designed to realize Fano resonances, including metallic nanoclusters $[9,10]$, individual plas- monic dolmen nanocavity [11], ring/disk cavities [12,13], and metamaterials and metasurfaces [14-16]. In particular, as an important geometry, waveguide-cavity stuctures are investigated at different platforms aiming for Fano resonance. Fano-type transmission phenomenona were observed in photonic crystal (PhC) waveguide-cavity systems $[17,18]$. The $\mathrm{PhC}$ waveguide is a line defect formed by removing a row of rods or air holes. The cavity is a point defect formed by reducing the radius of a single rod or removing an air hole. Fano profiles appear when partially reflecting elements are placed in the $\mathrm{PhC}$ waveguide. 
The physical origin of the Fano profile is the coupling of a discrete state (provided by cavity) to a continuum (provided by the waveguide with partially reflectance).

In addition to $\mathrm{PhC}$ waveguides, metal-dielectric-metal (MDM) waveguides are very attractive for researchers because they can support surface plasmon polaritons (SPPs) and allow for the control of light at the subwavelength scale. MDM waveguides provide an effective approach to chip-scale photonic components [19-22]. Fano resonances have been obtained in MDMbased waveguide-cavity coupled systems [23,24]. In recent years, multiple Fano resonances have aroused interest [25-27]. Compared with a single Fano resonance, multiple Fano resonances have more versatile and flexible applications, such as self-reference and multichannel sensing [25,26].

The generation of multiple Fano resonances is attributed to the existence of multiple discrete states. In most of the published papers, high-order modes are excited in the same cavity $[23,28]$. Discrete states are provided by different modes from the same cavity, thus the resulting multiple Fano resonances are connected to the different orders of the mode. As a result, the frequency interval of the Fano resonances is usually very large, ranging over hundreds of nanometers. Recently, multiple Fano resonances were achieved with the aid of different cavities that provided several discrete states $[26,29,30]$. For instance, two U-shaped resonators were designed in order to obtain independent dual resonances [30].

In this paper, multiple Fano resonances are investigated and demonstrated in MDM waveguide-cavity coupled systems. A symmetry-breaking ring cavity is proposed. Discrete states are obtained in the same cavity without exciting high-order cavity modes. Because of the equal mode order, the interval of the resonant frequency is reduced. The manipulation of Fano resonances is further discussed by adjusting the degree of asymmetry. More Fano peaks appear in the transmission spectrum after combining two symmetry-breaking cavities with different dimensions.

\section{Structure and Theory}

Figure 1 shows the schematic diagram of the MDM waveguide-cavity coupled system. The inset is the $3 \mathrm{D}$ view. The asymmetric cavity that we designed is an off-centered ring resonator (OCRR). $O$ and $O^{\prime}$ are the center of the outer and the inner circle, respectively. The distance between $O$ and $O^{\prime}$ is denoted by $d$. When $d=0$, the cavity is a regular ring. When $d \neq 0$, an off-centered ring with symmetry breaking is obtained. The deviation angle between the center connection and the $x$-axis is described by $\phi$. The distance $d$ and the angle $\phi$ are parameters that are closely related to the degree of asymmetry.

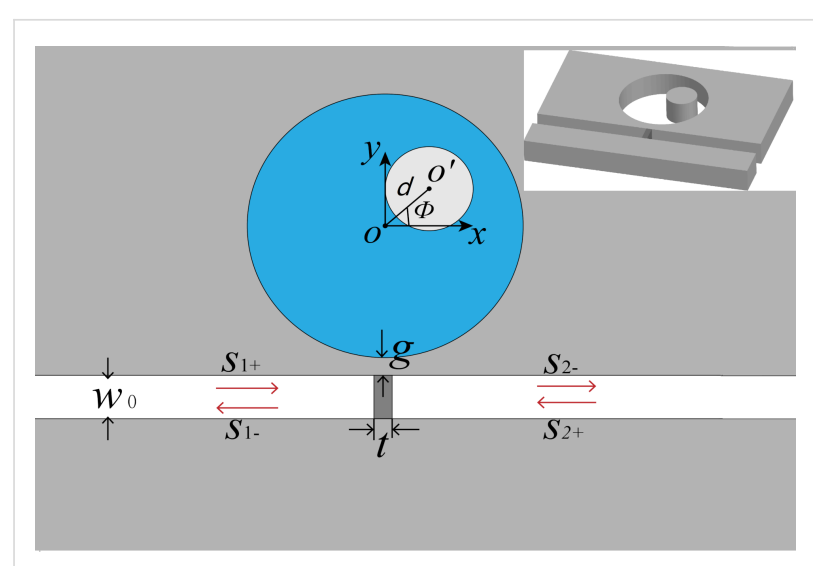

Figure 1: Schematic diagram of an off-centered ring resonator (OCRR) side-coupled to the MDM waveguide. The inset is the 3D view. The outer and inner radius of the OCRR are $R$ and $r$, respectively. The deviation of the center points is denoted by $d$ (i.e., $O O^{\prime}$ ). The deviation angle is described by $\phi$, which is the angle between the center connection and the $x$-axis. The width of waveguide is $W_{0}$. The thickness of the metal wall in the waveguide is $t$. The coupling distance between the disk resonator and the upper stub is $g$.

The radius of the outer and the inner ring cavity are $R$ and $r$, respectively. The OCRR is side-coupled to the waveguide with the gap $g$. The width of the MDM waveguide is $W_{0}$. A metal wall with the thickness $t$ is placed inside the MDM waveguide.

The grey parts in Figure 1 stand for metal $\left(\varepsilon_{\mathrm{m}}\right)$. Both the metal wall and the background metal are silver the complex relative permittivity of which is characterized by the Drude model,

$$
\varepsilon_{\mathrm{m}}=\varepsilon_{\infty}-\frac{\omega_{\mathrm{p}}^{2}}{\omega^{2}+\mathrm{i} \gamma \omega},
$$

where $\omega$ is the angular frequency of the incident light. The other parameters are $\varepsilon_{\infty}=3.7$, bulk plasma frequency $\omega_{\mathrm{p}}=1.38 \times 10^{16} \mathrm{~Hz}$, damping frequency $\gamma=2.73 \times 10^{13} \mathrm{~Hz}$. The dielectric in the waveguide is air. The ring resonator is filled with a dielectric with the constant $\varepsilon_{\mathrm{d}}$.

Temporal coupled-mode theory (CMT) is used to analyze the transmission characteristics [25,31]. For the studied waveguide-resonator coupled system, the coupling coefficients between ring resonator and input (output) waveguide are denoted by $\kappa_{1}\left(\kappa_{2}\right) . \theta_{1}\left(\theta_{2}\right)$ are the phase shift of the coupling coefficient between ring resonator and input (output) waveguide. The decay rate due to the internal loss in the resonators is $\kappa_{i}$. The amplitudes of the incoming and outgoing waves in the waveguide are denoted by $S_{i+}$ and $S_{i-}(i=1,2)$.

The time evolution of the normalized amplitude $a$ of ring resonator can be expressed as 


$$
\begin{aligned}
\frac{\mathrm{d} a}{\mathrm{~d} t}= & \left(j \omega_{0}-\kappa_{i}-\kappa_{1} / 2-\kappa_{2} / 2\right) a_{1} \\
& +e^{-j \theta_{1}} \sqrt{\kappa_{1}} S_{1+}+e^{-j \theta_{2}} \sqrt{\kappa_{2}} S_{2+} .
\end{aligned}
$$

According to energy conservation, the amplitudes of the incoming and the outgoing waves in coupled waveguides satisfy the following relationships:

$$
S_{2-}=t_{\mathrm{m}} S_{1+}-e^{-j \theta_{2}} \sqrt{\kappa_{2}} a,
$$

where $t_{\mathrm{m}}$ is the transmission coefficient for SPPs passing through the waveguide with a metal wall inside. It represents the direct coupling between input waveguide and output waveguide. If there is no metal wall in the waveguide $t_{\mathrm{m}}$ is almost equal to 1 . Equation 2 can then be rewritten as $S_{2-}=S_{1+}-e^{-j \theta_{2}} \sqrt{\kappa_{2}} a$. If the transmission is completely forbidden due to metal wall, then $t_{\mathrm{m}}=0$.

When an optical wave with frequency $\omega$ is launched only from the left port of the waveguide $\left(S_{2+}=0\right)$, the transmission of waveguide-resonator coupled system can be derived as:

$$
T=\left|\frac{S_{2-}}{S_{1+}}\right|^{2}=\left|t_{\mathrm{m}}-\frac{e^{j\left(\theta_{1}-\theta_{2}\right)} \sqrt{\kappa_{1}} \sqrt{\kappa_{2}}}{j\left(\omega-\omega_{0}\right)+\kappa_{i}+\left(\kappa_{1}+\kappa_{2}\right) / 2}\right|^{2} .
$$

If the width of the input waveguide is the same as the width of the output waveguide, then $\theta_{1}=\theta_{2}$ and $\kappa_{1}=\kappa_{2}$. When there is no metal wall (corresponding to $t_{\mathrm{m}}=1$ ), the transmission efficiency through the waveguide-resonator system is described by:

$$
T=\left|1-\frac{\kappa}{j\left(\omega-\omega_{0}\right)+\kappa_{i}+\kappa}\right|^{2}=\left|\frac{j\left(\omega-\omega_{0}\right)+\kappa_{i}}{j\left(\omega-\omega_{0}\right)+\kappa_{i}+\kappa}\right|^{2} .
$$

The above expression agrees with Equation 3 in [32], which deals with a waveguide with a side-coupled nanocavity. Equation 4 shows that at the resonance frequency $\omega_{0}$ the transmission reaches minimum: $T_{\min }=\left|\kappa_{i} /\left(\kappa_{i}+\kappa\right)\right|^{2}$. This means there would be a narrow transmission dip. If there is no internal loss $\kappa_{i}$, we have $T_{\min }=0$. When the transmission coefficient $t_{\mathrm{m}}$ is relatively low and closes to zero, Equation 3 can be expressed as:

$$
T=\left|-\frac{\kappa}{j\left(\omega-\omega_{0}\right)+\kappa_{i}+\kappa}\right|^{2} .
$$

Equation 5 shows that the maximum transmission $T_{\max }$ happens when $\omega=\omega_{0}: T_{\max }=\left|\kappa /\left(\kappa_{i}+\kappa\right)\right|^{2}$. The transmission spectrum has a peak around the resonance frequency $\omega_{0}$. If there is no internal loss $\kappa_{i}$, we have $T_{\max }=1$.

\section{Results and Discussion}

Numerical simulations were performed by using COMSOL Multiphysics. The width of the waveguide is $W_{0}=50 \mathrm{~nm}$, the gap between waveguide and resonator is $g=10 \mathrm{~nm}$. The outer and inner radius of ring are $R=155 \mathrm{~nm}$ and $r=55 \mathrm{~nm}$, respectively. The deviation angle is $\phi=0^{\circ}$ and the deviation distance is $d=0$.

Figure 2 shows the transmission spectra of the designed waveguide-resonator coupled system. Different values of the thickness of the metal wall, $t$, were studied. When a metal wall is placed in a MDM waveguide, the transmittance is low over a wide spectral range (green curve). The continuum state is provided by the waveguide with a metal wall inside (WgM). When a ring is side-coupled to an ordinary waveguide ( $\mathrm{Wg}+\mathrm{Ring}$ ), a narrow dip at $807 \mathrm{~nm}$ appears in the transmission spectrum (blue curve). This dip means that the discrete state can be provided by the side-coupled ring cavity. For the system consisting of a ring and a waveguide with inserted metal wall (WgM+Ring), a transmission peak appears at around $800 \mathrm{~nm}$ (red curve) in an asymmetric transmission profile. When metal thickness is $t=10 \mathrm{~nm}$, the asymmetric line shape is more obvious, as shown in Figure 2b. We consider this resonance a Fano resonance that arises from the interference of a resonant ring mode and a waveguide mode. From Figure 2, we infer that the position of the Fano peak is determined more strongly by the ring cavity.

In the following, the influence of the ring cavity on the Fano resonance is investigated. The evolution of the transmission spectra as a function of the inner radius is given in Figure 3a. The transmission peaks move to longer wavelengths with the increase of $r$. The inset shows the resonance wavelength $\lambda_{0}$ as a function of $r$. The resonance wavelength is proportional to $r$. This can be explained by the resonance function. The resonance wavelengths are determined by:

$$
\begin{aligned}
& \lambda_{0, m}=\operatorname{Re}\left(n_{\mathrm{eff}}\right) L_{\mathrm{eff}} / m, \\
& L_{\mathrm{eff}}=2 \pi R_{\mathrm{eff}}=\pi(R+r),
\end{aligned}
$$

where $L_{\text {eff }}$ is the effective length of ring resonator. $m$ is an integer corresponding to the mode order, and $n_{\text {eff }}$ is the effective index, which can be obtained from dispersion equation [33]. 

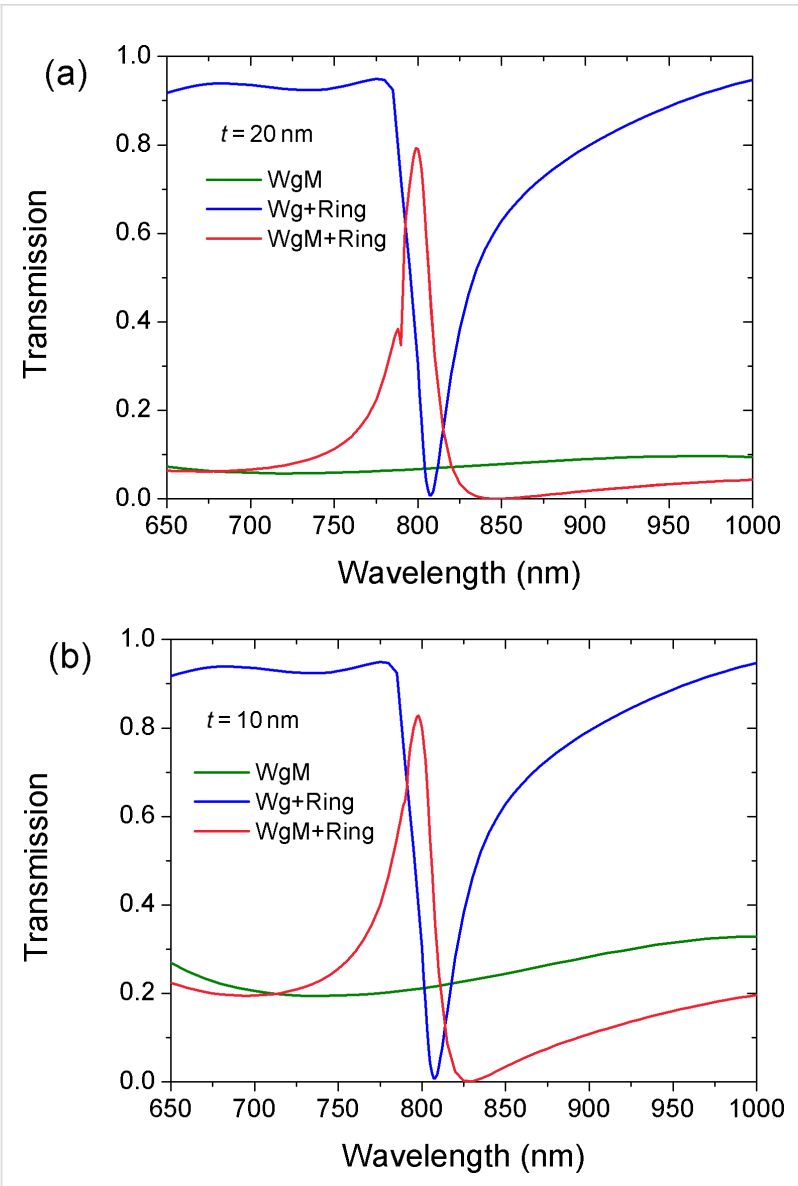

Figure 2: Transmission spectra for different structures. (a) $t=20 \mathrm{~nm}$, and (b) $t=10 \mathrm{~nm}$. The other parameters are $R=155 \mathrm{~nm}, r=55 \mathrm{~nm}$ and $\phi=0^{\circ}$.

Figure 4 presents the effective index $n_{\text {eff }}$ as a function of the wavelength for different ring widths $W$. It is observed that the larger the width $W$, the lower the effective index $n_{\text {eff }}$. Combining Equation 6 and Figure 4, we conclude that the resonance frequency of ring depends on the radii and the width of the ring. When the inner radius $r$ increases from 35 to $75 \mathrm{~nm}$, the effective length $L_{\text {eff }}$ becomes larger, and at the same time the ring width decreases from 120 to $80 \mathrm{~nm}$, which leads to bigger index $n_{\text {eff }}$. As a result, both $L_{\text {eff }}$ and $n_{\text {eff }}$ increase with the increase of radius $r$. According to Equation 6, the corresponding resonance wavelength will become longer. The simulation shows that the Fano peak has a red-shift, which is consistent with the analysis.

Figure $3 \mathrm{~b}$ shows the evolution of transmission as a function of the outer radius $R$. The inset shows the resonance wavelength $\lambda_{0}$ as a function of $R$. With the increase of the outer radius $R$ the transmission peak moves to longer wavelengths. Note that the red-shift in Figure $3 b$ is smaller compared with that in Figure 3a. We attribute this phenomenon to different impacts of $L_{\text {eff }}$ and $n_{\text {eff }}$ on the transmission. When outer radius $R$ increases
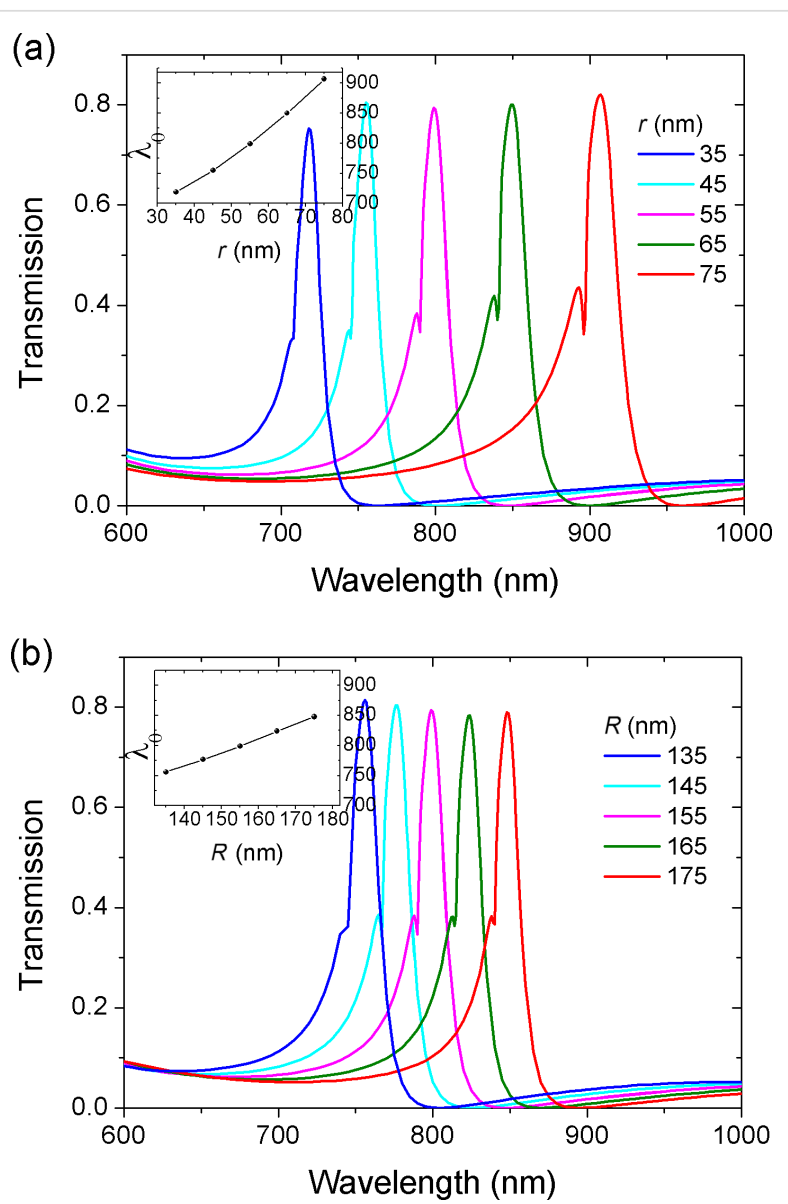

Figure 3: Transmission spectra at different structural parameters: (a) inner radius $r$, (b) outer radius $R$. The inset shows the resonance wavelength $\lambda_{0}$ as a function of radius $r$.

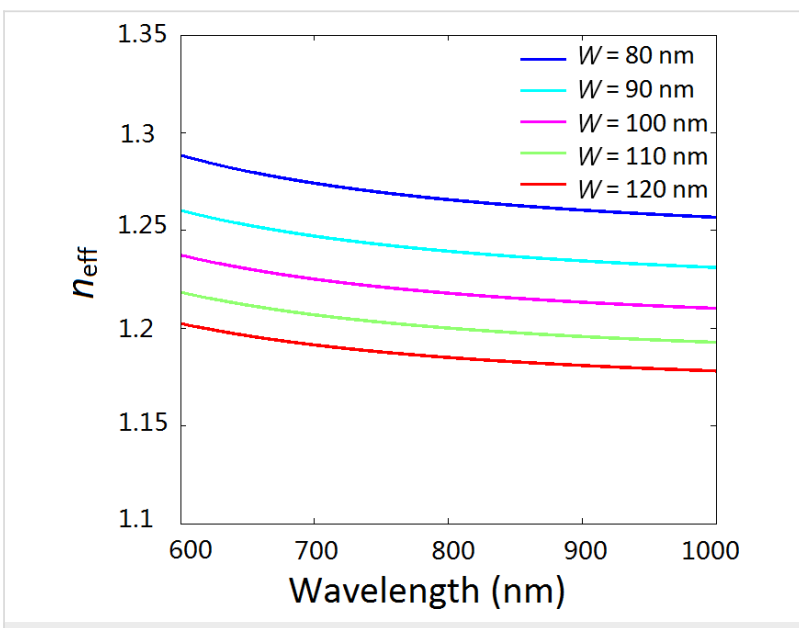

Figure 4: Effective refraction index $n_{\text {eff }}$ as a function of the wavelength at different ring widths $W$.

from 135 to $175 \mathrm{~nm}$, the ring width $W$ increases from 80 to $120 \mathrm{~nm}$. Bigger values of $W$ will result in smaller $n_{\text {eff. }}$ On the contrary, the effective length $L_{\mathrm{eff}}$ increases with $R$. The blue- 
shift of the resonance wavelength caused by decreasing $n_{\mathrm{eff}}$ will partially offset the red-shift of the resonance wavelength caused by increasing $L_{\text {eff }}$. This is the reason why the shift of the resonance peak in Figure $3 b$ is smaller than that in Figure $3 \mathrm{a}$.

We have demonstrated that the ring is the key component for controlling the resonant frequency. In the following, we break the symmetry of the ring resonator and investigate the impact of symmetry-breaking on the Fano resonance. The symmetrybreaking is introduced by deviating center points $O$ and $O^{\prime}$. When the deviation distance $d$ is greater than 0 , a regular ring becomes an off-centered ring. Figure 5a displays the transmission spectra at $d=80 \mathrm{~nm}$ with varying angle $\phi$. When $\phi$ is rotated counterclockwise, a new transmission peak 2 appears on the left side of the initial peak 1 . With the increase of $\phi$, the amplitude of peak 2 gradually increases while the amplitude of peak 1 gradually decreases. When $\phi$ reaches $90^{\circ}$, resonance 1 is totally suppressed and only resonance 2 exists. When $\phi$ is rotated clockwise from $0^{\circ}$ to $-90^{\circ}$, a similar phenomenon is observed (Figure 5c). A new resonance peak 2 appears and gradu- ally increases its amplitude. Meanwhile, peak 1 is gradually decreasing. We note that the line widths of peak 2 in Figure 5a and Figure $5 \mathrm{~b}$ differ. The electric field distributions for peak 1 and 2 at $\phi=45^{\circ}$ and $-45^{\circ}$ are shown in Figure $5 \mathrm{~b}$ and Figure $5 \mathrm{~d}$, respectively. It is observed that peak 1 and peak 2 belong to the first mode order but their field patterns have different symmetry. It is the symmetry-breaking of the ring that leads to the change in the field distribution. The redistribution of field intensity would affect the coupling strength between ring resonator and waveguide. As a result, the linewidths of the resonance peaks differ.

Figure 6 shows the evolution of the transmission spectra at different deviation distances $d$. For clarity, only the angles $\phi$ are plotted. A new resonance peak 2 can be observed when $d$ equals 40 and $80 \mathrm{~nm}$. Comparing Figure 6a with Figure 6b, we find that as $d$ increases, resonance 1 undergoes a red-shift while resonance 2 undergoes a blue-shift. The opposite shift leads to an increased wavelength interval between the two peaks at larger $d$. The reason is attributed to a greater asymmetry caused by a larger deviation distance $d$.
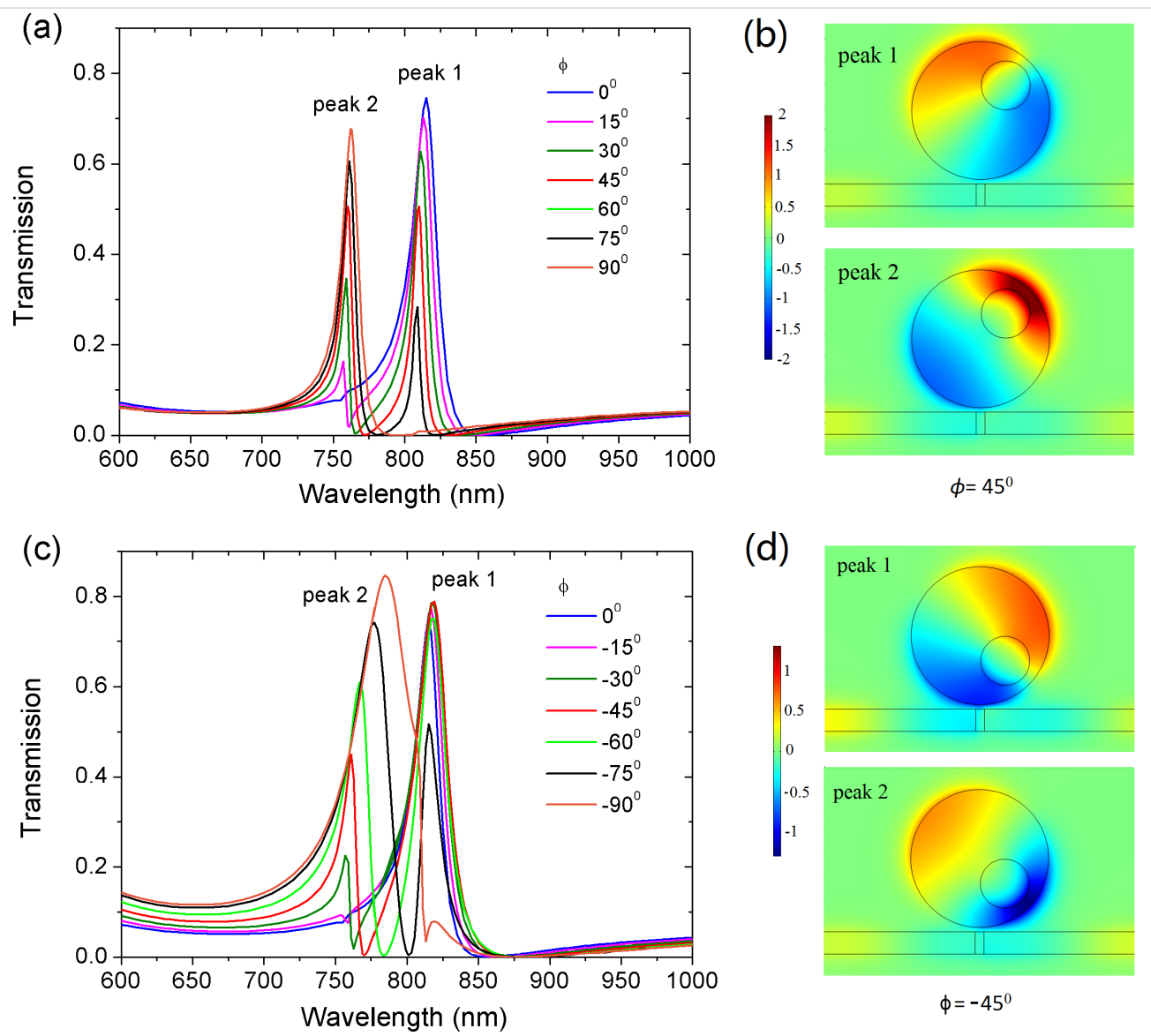

Figure 5: The evolution of transmission spectra with angle $\phi$ varying (a) from $0^{\circ}$ to $90^{\circ}$, and (c) from $0^{\circ}$ to $-90^{\circ}$. The electric field distribution for peak I and II at (b) $\phi=45^{\circ}(810 \mathrm{~nm}$ and $760 \mathrm{~nm})$, and (d) $\phi=-45^{\circ}(819 \mathrm{~nm}$ and $761 \mathrm{~nm})$. The other parameters are $R=155 \mathrm{~nm}, r=55 \mathrm{~nm}$, and the deviation distance $d=80 \mathrm{~nm}$. 

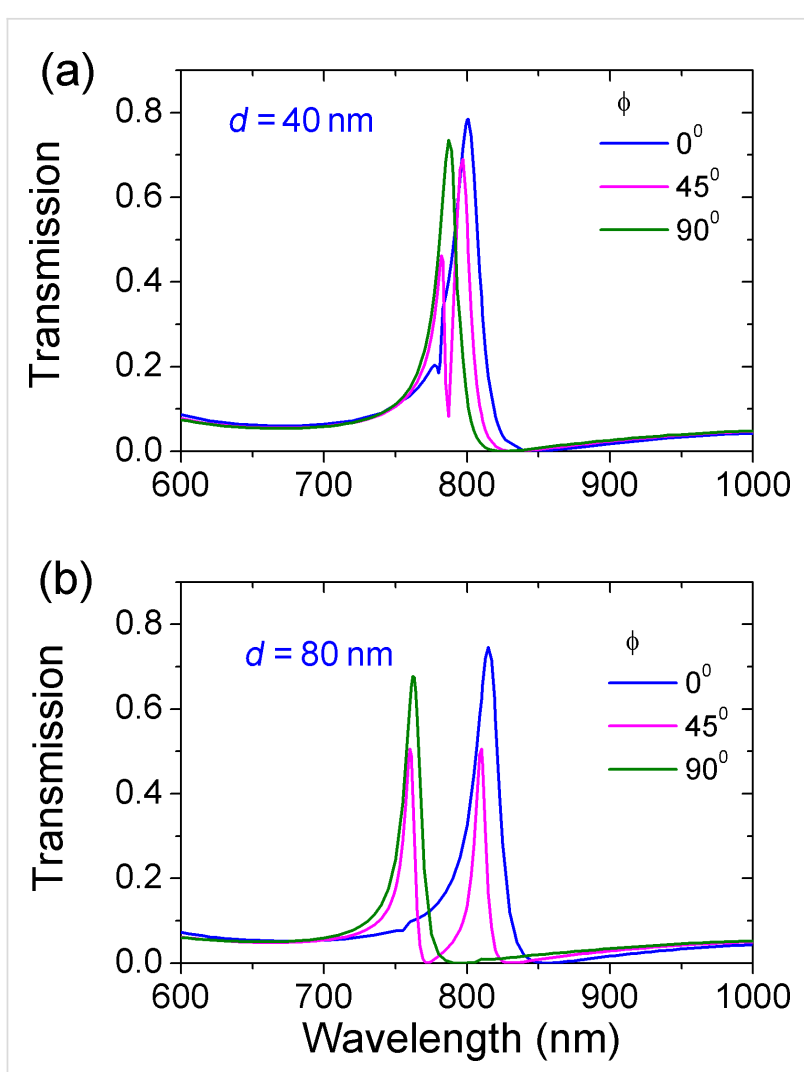

Figure 6: The evolution of transmission spectra with angle $\phi$. The deviation distance is (a) $d=40 \mathrm{~nm}$ and (b) $d=80 \mathrm{~nm}$. The other parameters are $R=155 \mathrm{~nm}$ and $r=55 \mathrm{~nm}$.

We have demonstrated in Figure 5 and Figure 6 that the dual resonances can be obtained by breaking the symmetry of ring resonator. Moreover, the resonance frequency and the number of peaks can be tailored through altering the degree of asymmetry, i.e., the deviation distance $d$ and the deviation angle $\phi$.

Now, another ring is placed at the other side of the waveguide with the gap distance $g=10 \mathrm{~nm}$. The outer and the inner radius of the second ring are $R_{2}=155 \mathrm{~nm}$ and $r_{2}=75 \mathrm{~nm}$, respectively. The deviation angle is $\phi_{2}=-45^{\circ}$ and the deviation distance is $d_{2}=55 \mathrm{~nm}$. Figure 7 shows the transmission spectra when the deviation angle $\phi$ of the first ring changes. Different deviation distances $d$ are considered here. Two new peaks located at 880 and $925 \mathrm{~nm}$ are observed in addition to the original resonance peaks around $800 \mathrm{~nm}$. For clarity, we refer to the new emergent resonance peaks as band II and to the initial resonance peaks as band I. In the presence of two resonators, the resonances of band I in Figure 7 show the same characteristic as the resonances in Figure 6. At $\phi=0^{\circ}$ and $\phi=90^{\circ}$, only one peak is excited. At $\phi=45^{\circ}$ both peak 1 and peak 2 are excited. Furthermore, the larger the distance $d$, the more distinct is the wavelength interval between peak 1 and 2 . However, the reso- (a)
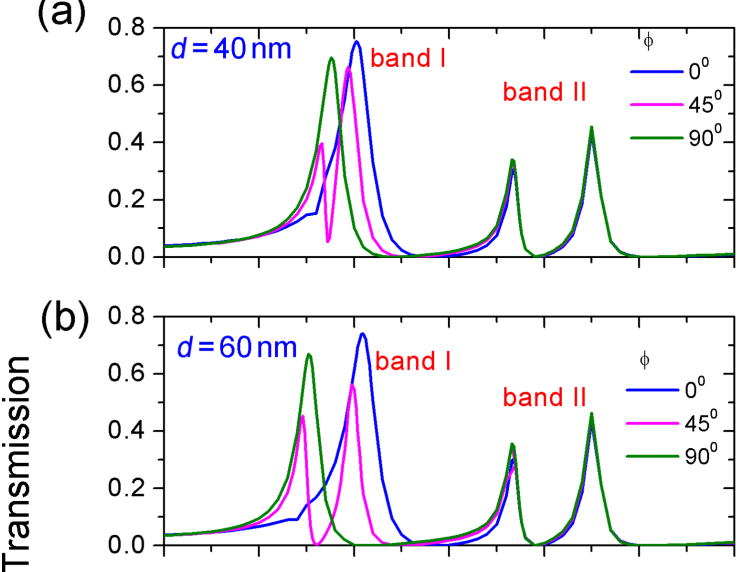

(c)

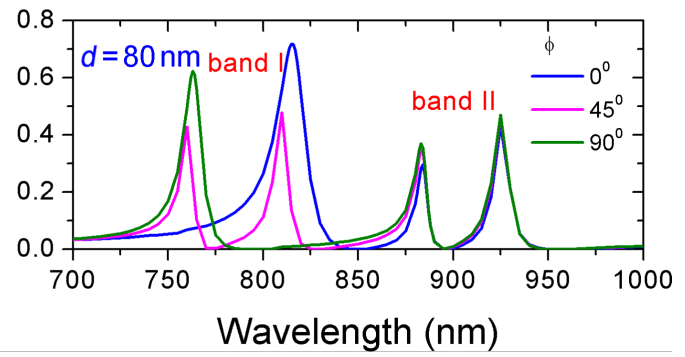

Figure 7: The evolution of transmission spectra as a function of the deviation angle $\phi$ of the first ring. The deviation distances are (a) $d=40 \mathrm{~nm}$, (b) $d=60 \mathrm{~nm}$, and (c) $d=80 \mathrm{~nm}$. The other parameters of the initial ring are $R=155 \mathrm{~nm}$ and $r=55 \mathrm{~nm}$. The deviation angle is $\phi=45^{\circ}$ and deviation distance is $d=80 \mathrm{~nm}$. The parameters for of the second ring are $R_{2}=155 \mathrm{~nm}, r_{2}=75 \mathrm{~nm}, d_{2}=55 \mathrm{~nm}$, and $\phi_{2}=-45^{\circ}$.

nances of band II keep unchanged as $\phi$ varies. This different behavior demonstrates that band I stems from the coupling of the first ring and the waveguide. Therefore, band I depends on parameters of the first ring and is not influenced by the second ring.

The impact of deviation angle $\phi_{2}$ of the second ring on the transmission was also investigated. The transmission spectra for varying $\phi_{2}$ at different deviation distances $d_{2}$ are plotted in Figure 8. A similar phenomenon is observed. One or two resonances of band II appear as the deviation angle $\phi_{2}$ changes. Furthermore, the wavelength interval between resonance peaks 3 and 4 gets larger with increased $d$. However, the resonances of band I are independent of $\phi_{2}$. This behavior indicates that the resonances of band II originate from the coupling of the second ring and the waveguide.

From Figure 7 and Figure 8, we know that the resonances of band I is determined by the first ring and the resonances of band II depend on the second ring. This different dependence provides a good opportunity to separately tune multiple Fano resonances. Figure 9 shows the transmission spectra with different parameters of the two ring resonators. The numbers in each 
(a)
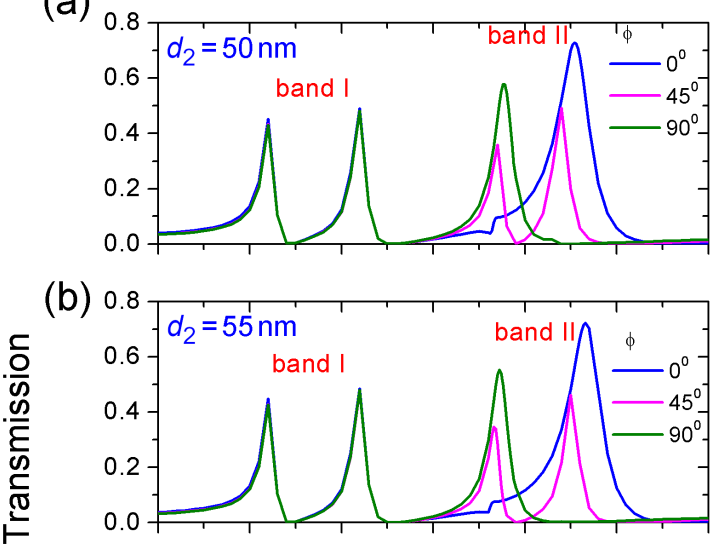

(c)

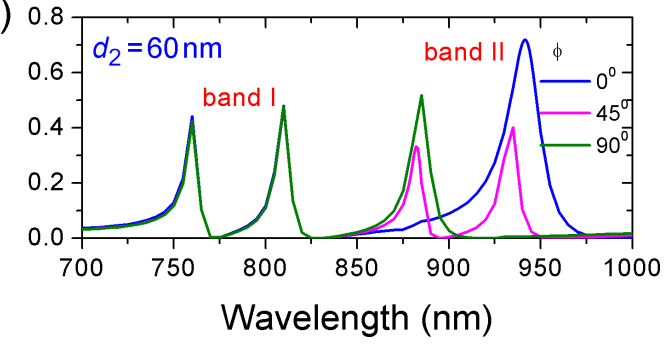

Figure 8: The evolution of transmission spectra as a function of the deviation angle $\phi_{2}$ of the second ring. The deviation distance $d_{2}$ is (a) $d_{2}=50 \mathrm{~nm}$, (b) $d_{2}=55 \mathrm{~nm}$, (c) $d_{2}=60 \mathrm{~nm}$. The other parameters of second ring are $R_{2}=155 \mathrm{~nm}, r_{2}=75 \mathrm{~nm}$. The parameters for initial ring are $R=155 \mathrm{~nm}, r=55 \mathrm{~nm}, d=80 \mathrm{~nm}, \phi=45^{\circ}$. subfigure stand for the value of $\left(d[\mathrm{~nm}], \phi\left[{ }^{\circ}\right]\right)$ and $\left(d_{2}[\mathrm{~nm}]\right.$, $\left.\phi_{2}\left[{ }^{\circ}\right]\right)$. The outer and inner radii are constant. $R=155 \mathrm{~nm}$, $r=55 \mathrm{~nm}, R_{2}=155 \mathrm{~nm}$, and $r_{2}=75 \mathrm{~nm}$. It is seen that dual, triple, and even quadruple Fano-type transmissions are obtained by separately adjusting the deviation distance and deviation angle of each off-centered ring. In particular, there are triple peaks in Figure 9b-e but their resonant frequencies and frequency interval are not the same. This shows that multiple resonances can be arbitrarily tailored by changing the degree of asymmetry.

We have demonstrated that the radius of the rings is a key factor for Fano resonance in Figure 2 and Figure 3. The impact of the radius on multiple Fano resonances in the presence of two rings is further investigated. Figure 10 shows the evolution of transmission spectra as a function of the inner radii $r$ and $r_{2}$. Fano resonant peaks undergo a red-shift with the increase of the inner radii. Band I is most affected by $r$, and band II is most affected by $r_{2}$. Multiple resonances can be independently tuned by changing the inner radii. Thus, one can regulate the frequency interval of resonance between band I and band II. Likewise, we find that the outer radius has an effect on the resonant frequency. In Figure 9 and Figure 10, we demonstrate that Fano resonances can be flexibly controlled by the structural parameters $(R, r, d, \phi)$ and $\left(R_{2}, r_{2}, d_{2}, \phi_{2}\right)$. These results are a great benefit to tunable integrated photonic devices.
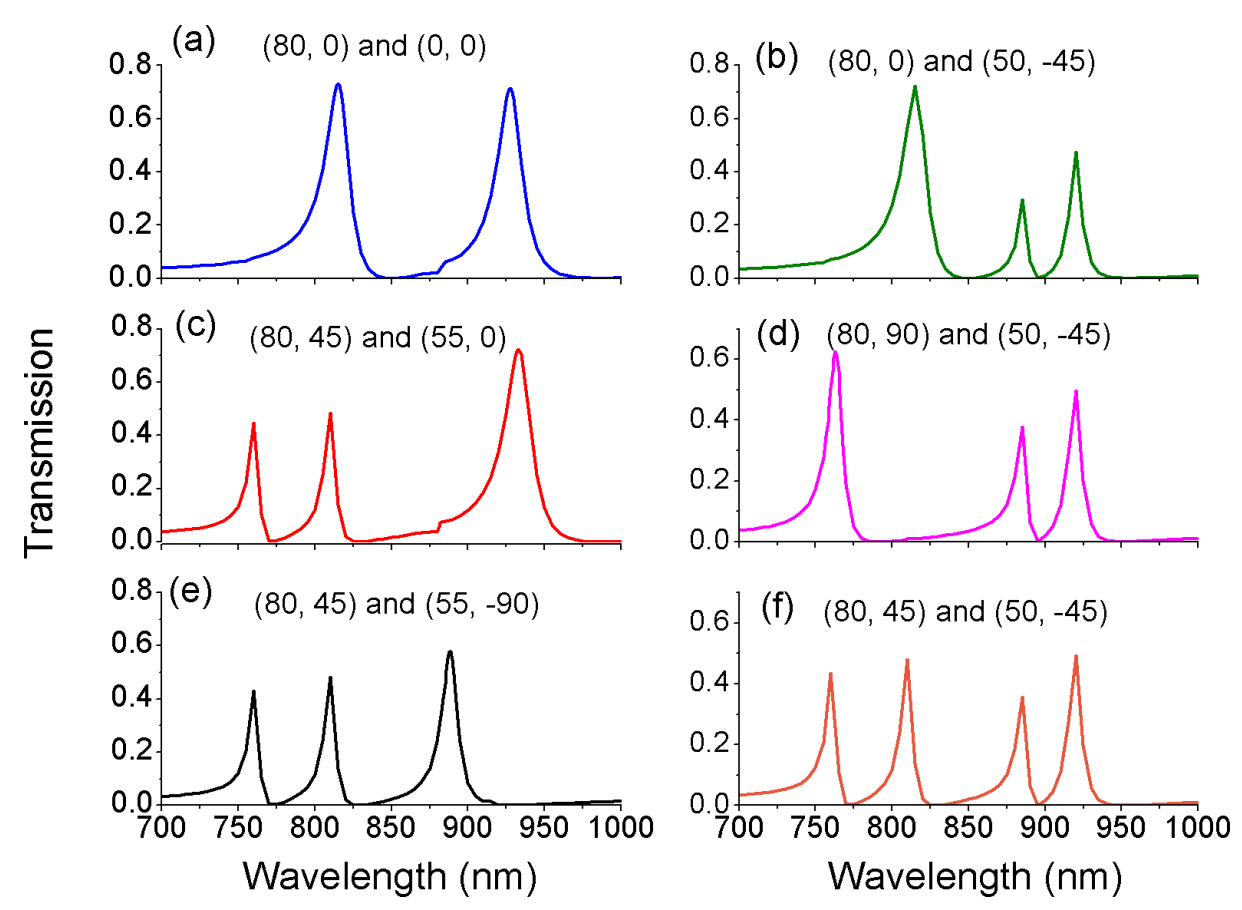

Figure 9: The evolution of transmission spectra with different parameters of two off-centered rings. $\left(d[\mathrm{~nm}], \phi\left[{ }^{\circ}\right]\right)$ and $\left(d_{2}[\mathrm{~nm}], \phi_{2}\left[{ }^{\circ}\right]\right)$ are $(\mathrm{a})(80,0)$ and $(0,0)$; (b) $(80,0)$ and $(50,-45)$; (c) $(80,45)$ and $(55,0)$; (d) $(80,90)$ and $(50,-45)$; (e) $(80,45)$ and $(55,-90)$; (f) $(80,45)$ and (50, -45). 


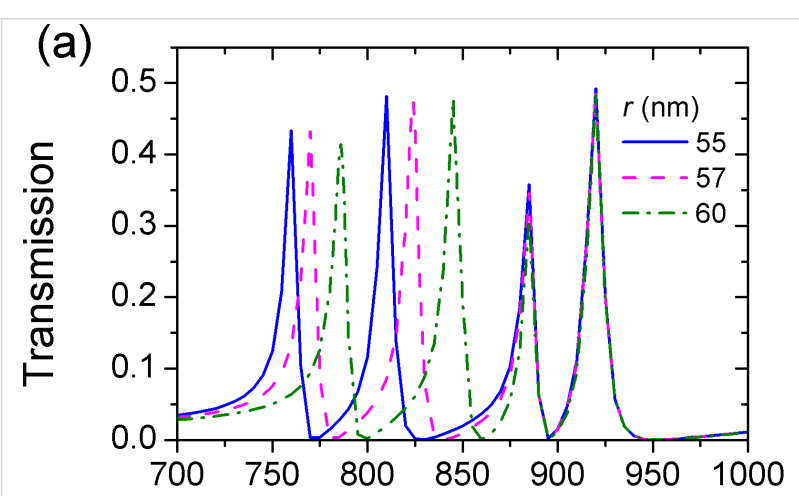

(b)

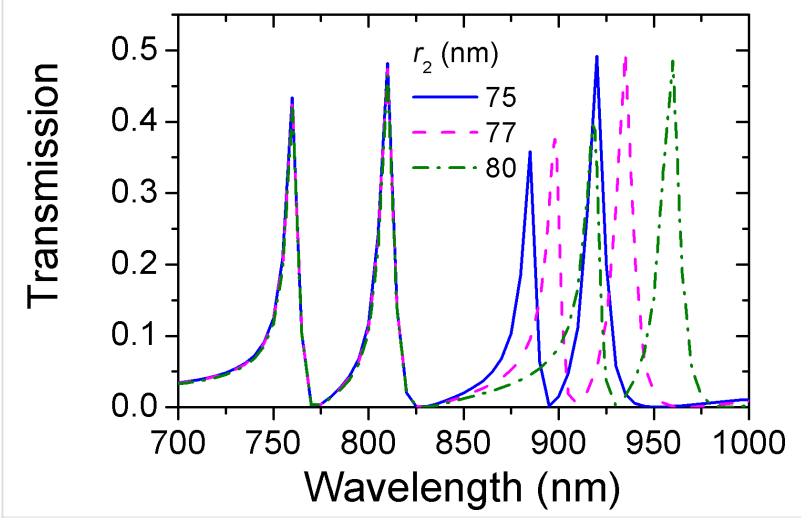

Figure 10: The evolution of transmission spectra with different radii. (a) Different values of $r$ at $r_{2}=75 \mathrm{~nm}$, (b) different values of $r_{2}$ at $r=55 \mathrm{~nm}$. The fixed parameters of the first ring are $R=155 \mathrm{~nm}$, $d=80 \mathrm{~nm}$, and $\phi=45^{\circ}$. The fixed parameters of the second ring are $R_{2}=155 \mathrm{~nm}, d_{2}=50 \mathrm{~nm}$, and $\phi_{2}=-45^{\circ}$.

\section{Conclusion}

We have demonstrated multiple Fano resonances in a system in which off-centered ring resonators are coupled to a waveguide. By breaking the symmetry of the ring resonators, new resonant modes that are not obtained with regular concentric ring resonators are excited. Degrees of asymmetry are adjusted by changing the deviation distance and deviation angle of the asymmetric ring resonators. Dual, triple and even quadruple Fano-type transmissions are arbitrarily tailored. The frequency interval of the multiple Fano resonances can be tuned. The results may provide good guidance for designing flexibly tunable integrated photonic devices.

\section{Acknowledgements}

This work was supported by the National Natural Science Foundation of China (11104200) and Natural Science Foundation of Tianjin (18JCYBJC17000).

\section{ORCID ${ }^{\circledR}$ iDs}

Kun Ren - https://orcid.org/0000-0003-4971-789X Qun Han - https://orcid.org/0000-0002-8139-8617

\section{Preprint}

A non-peer-reviewed version of this article has been previously published as a preprint doi:10.3762/bxiv.2019.50.v1

\section{References}

1. Fano, U. Phys. Rev. 1961, 124, 1866-1878. doi:10.1103/physrev.124.1866

2. Luk'yanchuk, B.; Zheludev, N. I.; Maier, S. A.; Halas, N. J.; Nordlander, P.; Giessen, H.; Chong, C. T. Nat. Mater. 2010, 9 , 707-715. doi:10.1038/nmat2810

3. Huang, C.-Y.; Chang, H. C. IEEE Photonics J. 2019, 11, 4800208. doi:10.1109/jphot.2018.2877323

4. Wu, C. H.; Khanikaev, A. B.; Adato, R.; Arju, N.; Yanik, A. A.; Altug, H.; Shvets, G. Nat. Mater. 2012, 11, 69-75. doi:10.1038/nmat3161

5. Chen, J.; Li, Z.; Li, J.; Gong, Q. Opt. Express 2011, 19, 9976-9985. doi:10.1364/oe.19.009976

6. Wang, L.; Qu, J.; Song, J.; Xian, J. Plasmonics 2017, 12, 1145-1151. doi:10.1007/s11468-016-0369-0

7. Zhao, W.; Leng, X.; Jiang, Y. Opt. Express 2015, 23, 6858-6866. doi:10.1364/oe.23.006858

8. Manjappa, M.; Srivastava, Y. K.; Cong, L.; Al-Naib, I.; Singh, R. Adv. Mater. (Weinheim, Ger.) 2017, 29, 1603355. doi:10.1002/adma.201603355

9. Lassiter, J. B.; Sobhani, H.; Fan, J. A.; Kundu, J.; Capasso, F.; Nordlander, P.; Halas, N. J. Nano Lett. 2010, 10, 3184-3189. doi: $10.1021 / \mathrm{nl} 102108 \mathrm{u}$

10. Nguyen, T. K.; Le, T. D.; Dang, P. T.; Le, K. Q. J. Opt. Soc. Am. B 2017, 34, 668-672. doi:10.1364/josab.34.000668

11. Verellen, N.; Sonnefraud, Y.; Sobhani, H.; Hao, F.; Moshchalkov, V. V.; Dorpe, P. V.; Nordlander, P.; Maier, S. A. Nano Lett. 2009, 9, 1663-1667. doi:10.1021/n19001876

12. Hao, F.; Sonnefraud, Y.; Dorpe, P. V.; Maier, S. A.; Halas, N. J.; Nordlander, P. Nano Lett. 2008, 8, 3983-3988. doi:10.1021/nl802509r

13. Cetin, A. E.; Altug, H. ACS Nano 2012, 6, 9989-9995. doi:10.1021/nn303643w

14. Huang, W.-X.; Guo, J.-J.; Wang, M.-S.; Zhao, G.-R. Phys. Lett. A 2017, 381, 909-912. doi:10.1016/j.physleta.2017.01.005

15. Campione, S.; Liu, S.; Basilio, L. I.; Warne, L. K.; Langston, W. L.; Luk, T. S.; Wendt, J. R.; Reno, J. L.; Keeler, G. A.; Brener, I.; Sinclair, M. B. ACS Photonics 2016, 3, 2362-2367. doi:10.1021/acsphotonics.6b00556

16. Muhammad, N.; Ouyang, Z.; Liu, Q.; Tang, X.; Deng, Z.-L.; Daud Khan, A. J. Mater. Sci. 2019, 54, 6301-6309. doi:10.1007/s10853-018-03228-3

17. Fan, S. Appl. Phys. Lett. 2002, 80, 908-910. doi:10.1063/1.1448174

18. Zhao, Y.; Qian, C.; Qu, K.; Tang, J.; Sun, Y.; Jin, K.; Xu, X. Sci. Rep. 2016, 6, 33645. doi:10.1038/srep33645

19. Abdulnabi, S. H.; Abbas, M. N. J. Nanophotonics 2019, 13, 016009. doi:10.1117/1.jnp.13.016009

20. Lu, H.; Yue, Z.; Zhao, J. Opt. Commun. 2018, 414, 16-21. doi:10.1016/j.optcom.2017.12.083

21. Dong, L.; Xu, X.; Li, C.; Guo, Y.; Sun, K.; Ding, Y. Opt. Commun. 2018, 410, 751-755. doi:10.1016/j.optcom.2017.11.048

22. Yang, J.; Song, X.; Yang, S.; Cui, L.; Yu, L. J. Phys. D: Appl. Phys. 2017, 50, 325107. doi:10.1088/1361-6463/aa77a2

23. Ren, X.; Ren, K.; Cai, Y. Appl. Opt. 2017, 56, H1-H9. doi:10.1364/ao.56.0000h1

24. Tang, Y.; Zhang, Z.; Wang, R.; Hai, Z.; Xue, C.; Zhang, W.; Yan, S. Sensors 2017, 17, 784. doi:10.3390/s17040784 
25. Ren, K.; Ren, X.; He, Y.; Han, Q. Beilstein J. Nanotechnol. 2019, 10, 247-255. doi:10.3762/bjnano.10.23

26. Guo, Z.; Wen, K.; Hu, Q.; Lai, W.; Lin, J.; Fang, Y. Sensors 2018, 18, 1348. doi:10.3390/s18051348

27. Chen, Z.; Li, H.; He, Z.; Xu, H.; Zheng, M.; Zhao, M. Appl. Phys. Express 2017, 10, 092201. doi:10.7567/apex.10.092201

28. Li, C.; Li, S.; Wang, Y.; Jiao, R.; Wang, L.; Yu, L. IEEE Photonics J. 2017, 9, 4801509. doi:10.1109/jphot.2017.2763781

29. Shi, X.; Ma, L.; Zhang, Z.; Tang, Y.; Zhang, Y.; Han, J.; Sun, Y. Opt. Commun. 2018, 427, 326-330. doi:10.1016/j.optcom.2018.06.042

30. Ren, X.; Ren, K.; Ming, C. Sensors 2018, 18, 1376. doi:10.3390/s18051376

31. Deng, Y.; Cao, G.; Wu, Y.; Zhou, X.; Liao, W. Plasmonics 2015, 10 , 1537-1543. doi:10.1007/s11468-015-9971-9

32. Lu, H.; Liu, X.; Gong, Y.; Wang, L.; Mao, D. Opt. Commun. 2011, 284, 2613-2616. doi:10.1016/j.optcom.2011.01.046

33. Dionne, J. A.; Sweatlock, L. A.; Atwater, H. A.; Polman, A. Phys. Rev. B 2006, 73, 035407. doi:10.1103/physrevb.73.035407

\section{License and Terms}

This is an Open Access article under the terms of the Creative Commons Attribution License (https://creativecommons.org/licenses/by/4.0). Please note that the reuse, redistribution and reproduction in particular requires that the authors and source are credited.

The license is subject to the Beilstein Journal of Nanotechnology terms and conditions: (https://www.beilstein-journals.org/bjnano)

The definitive version of this article is the electronic one which can be found at: doi:10.3762/bjnano.10.236 